LAW RENCE LIVERMORE N A T IO N A L LABORATORY

Planning Electron cloud/Gas desorption activities in the HIF-VNL during FY06

A. W. Molvik

September 23, 2005 
This document was prepared as an account of work sponsored by an agency of the United States Government. Neither the United States Government nor the University of California nor any of their employees, makes any warranty, express or implied, or assumes any legal liability or responsibility for the accuracy, completeness, or usefulness of any information, apparatus, product, or process disclosed, or represents that its use would not infringe privately owned rights. Reference herein to any specific commercial product, process, or service by trade name, trademark, manufacturer, or otherwise, does not necessarily constitute or imply its endorsement, recommendation, or favoring by the United States Government or the University of California. The views and opinions of authors expressed herein do not necessarily state or reflect those of the United States Government or the University of California, and shall not be used for advertising or product endorsement purposes.

This work was performed under the auspices of the U.S. Department of Energy by University of California, Lawrence Livermore National Laboratory under Contract W-7405-Eng-48. 


\section{Planning Electron cloud/Gas desorption activities in the HIF-VNL during FY06 Art Molvik \\ 9/20/05}

The Heavy-Ion Fusion (HIF) group, under the DOE Office of Fusion Energy Science (OFES) funding, has been carrying out studies of e-cloud and gas primarily for our own needs. During this effort we have developed unique experimental and simulation tools that we believe have broader applications.

To a limited degree, as part of OFES' charter, we can pursue basic science for plasma and accelerator research and can also pursue issues of interest in high energy physics and other areas of accelerator research. We would appreciate your suggestions on specific needs that you have for which we might be able to make contributions towards understanding and mitigation. The following list of potential tasks provides a guide to our capabilities, plus some directions that we are considering; they are designed around our facilities, but we are open to collaborating at other sites. We will be firming up our plans after funding is set for the year - we currently expect that to happen in late October.

The following list of tasks for FY06 assumes significant restoration of funds by Congress to a similar level as in FY05. Each area would be studied with coordinated experimental and simulation efforts. Most of these tasks deal with electron or gas issues, the last few are more general high-brightness beam issues.

\section{Publish papers on recent work}

PRL: Gas desorbs due to electronic sput (with GSI/CERN) Molvik

Nov. '05

PRSTAB?: Scaling e- emission with $\mathrm{dE} / \mathrm{dx} \_$electronic $\quad$ Kireeff Covo Nov '05?

PRSTAB?: Desorbed gas velocity measurement Bieniosek/Molvik Dec. '05

PRSTAB?: Simulate reconstructed beam vs Z-ing

Seidl

Dec '05?

\section{Investigate new areas}

\section{A. Accumulation of electrons}

Using a retarding potential analyzer to measure the energy of expelled ions (from beam ionization of gas), we observe the beam potential decreasing in time, and decreasing further when we add electrons. This enables us to determine electron accumulations. We can do these experiments where we minimize all sources but one out of: gas ionization, beam-tube emission, and end-wall emission. We can then measure the accumulation from each source separately and determine electron trapping efficiencies. 


\section{B. Effect of electrons on beam}

We measure the electron accumulation, as in (A) above, vary it for each source, and measure the beam phase space with slit scanners. We expect the threshold for observing electron effects on the beam to depend on the source. [Electrons from gas ionization map out the beam profile and are predicted have the least affect, compared with the other sources where electrons extend to the beam tube. See R. Cohen, et al., PRSTAB 7, 124201 (2004).]

\section{Transport of electrons through quadrupole magnets}

We have measured and simulated the drift velocity of electrons through quadrupole magnets, finding good agreement. We have varied the potential of the end wall with + bias so primary electrons cannot reach the beam tube to generate secondary electrons we observed some effects that we need to do more work to understand. We are planning to insert a biased filament to inject controlled numbers of electrons, then study their accumulation and transport through magnets. We can have a negatively-biased-ring electrode outside both ends of the quadrupole magnets - this with the positive beam potential creates electrostatic confinement for electrons, enabling us to study losses through a negative suppressor electrode as a function of suppressor voltage or electron accumulation.

\section{Study electron drift instability in last quad. $\left(\mathbf{n}_{\mathrm{e}} \sim \mathbf{n}_{\mathrm{b}}\right)$}

This is a rather spectacular several-MHz instability of drifting electrons, seen in detail in simulations. Simulations of the clearing electrode currents agree very well with measured oscillations in the current to the last clearing electrode. I'll be talking about progress towards understanding this at the APS-DPP in Denver, Oct. 24-28, 2005. This instability isn't normally a problem: we create it by turning the suppressor off to flood the last magnet with electrons from the end wall. The high electron densities also create the " $Z$ " shaped X-X' phase space seen under these conditions.

\section{E. Study removal of electrons with clearing electrodes}

These have been demonstrated to reduce electron accumulation. When off, we have an electron confinement device as discussed under $(\mathrm{C})$. These are a prime diagnostic to determine whether we have eliminated all electron sources.

\section{F. Desorbed gas velocity measurements}

We have a good set of measurements from a gated visible-light camera viewing beamexcitation of gas desorbed by the beam from a stainless steel plate. These show gas velocities of up to several $\mathrm{mm} /$ microsec. We can obtain the gas velocity distribution from these data. We have separately used a residual gas analyzer (RGA) to measure that $\mathrm{H}_{2}$ is the dominant desorbed gas species $\left(\mathrm{H}_{2} \mathrm{O}\right.$ is the dominant background gas component). We could use an optical spectrometer and gated camera to measure the velocity of each gas species - but that requires a significant effort that we have not yet committed ourselves into.

These diagnostics could also be absolutely calibrated to determine first pass desorption coefficients. These could then measure desorption from NEG or other pumping surfaces, 
where significant desorption could occur, but over several bounces of the gas off walls, enough is pumped by the NEG to greatly reduce any transient pressure rise.

\section{G. Electron accumulation with solenoid transport}

It is well known that solenoids, added to drift regions, suppress e-clouds. I am not aware of anyone using solenoids for beam transport and studying the accumulation of electrons. We plan a first effort next Jan.-April, 2006.

\section{H. Possible two-stream instability}

Some simulations have shown the presence of energetic electrons in the last quadrupole magnet. We haven't measured electron energies in that location. If confirmed, this would seem to indicate instabilities with periods less than the several ns bounce time of electrons. We could study this further both experimentally and in simulations.

\section{New shorter quad magnets to provide smooth lattice with low phase advance} New magnets would avoid jumps in lattice period that causes beam pathologies independent of e- as shown with reconstructed beam simulations. This would allow for a clearer distinction between dynamical effects due to e-cloud, vs non-e-cloud (those due to initial beam distributions, depressed and undepressed phase advance and lattice transitions). The present thinking is to build a prototype magnet in FY06, with the intention of fabricating more as funding allows.

The HCX vacuum system achieves high-vacuum with "O" ring seals. We could build new magnets and a new diagnostics end tank with ultra-high-vacuum (UHV) construction. The magnets would provide a gas flow impedance to rest of HCX, allowing $\mathrm{UHV}$ in the end tank and to some degree in the last few magnets, increasing the relevance to other accelerators.

\section{J. Beam head/tail control with a correction induction core.}

We will model this first, to design the shape of the acceleration waveform that would most benefit beam head control. We will schedule availability of the programmable waveform induction cell with the Neutralized Drift Compression Experiment (NDCX), that is currently using it to achieve a factor of 50 longitudinal pulse compression. It should allow us to make head/tail scraping either worse or better. Providing poorer head control with significant envelope scraping of the beam tube could generate copious electrons that would be trapped by the rising beam potential, for study of that source. Improved head control should eliminate this source (currently believed to be a small effect on electron accumulation in HCX). Tail control would be of less interest for HIF power plants (with $\sim 0.2 \mathrm{~s}$ between pulses), but could be a useful tool in enabling more diagnostics to work - particularly those that measure electrons, as they are detrapped by the falling beam potential at the end of the pulse, have to deal with additional electrons being generated at the same time by the beam tail scraping the wall.

\section{K. Revisit electrostatic quadrupole (ESQ) transport}

ESQ transport with a high fill factor (ratio of beam major radius to beam tube radius) was reported by L. Prost in PRSTAB 8, 020101 (2005). We have very good RMS beam 
parameter agreement between envelope models and experiment. The objective now is to try for consistent experiment and simulation with regard to phase space and configuration space details, and (perhaps) to gain predictive capability for halos. We need closely spaced data before and after the 10 ESQs, taken under same conditions at closely spaced times. 